\title{
Custom designed dynamic videokeratometer
}

\author{
David Mas ${ }^{\mathrm{a} *}$, Malgorzata A. Kowalska ${ }^{\mathrm{b}}$, Julian Espinosa ${ }^{\mathrm{a}}$ and Henryk T. Kasprzak ${ }^{\mathrm{b}}$ \\ ${ }^{a}$ Dept. Optica, Universidad de Alicante, c/ Alicante, s.n. 03690, San Vicente del Raspeig, Alicante Spain; ${ }^{b}$ Inst. Physics, \\ Wroclaw University of Technology, Wybrzeze Wyspianskiego 27, 50-370 Wroclaw, Poland
}

\begin{abstract}
Human eye is a complex dynamic system that undergoes fine rotations and deformations. Usually information about the eye globe deformation and micromovements are averaged and lost when using commercial measuring devices, although they provide important information about the eye's physiology and the visual process. We have constructed a dynamic topographer from an existing Placido projection head and a video camera. The algorithms of calibration, data processing and topographic reconstruction are also explained in this paper. With our system, the CCD parameters and the algorithms can be controlled offering many possibilities for eye researchers. Obtained results show that the system is reliable for measuring eye dynamics. Some applications of the device are also outlined.
\end{abstract}

Keywords: dynamic videokeratometry; corneal topography; image processing

\section{Introduction}

Computerized videokeratometry has become a standard tool for measuring the corneal topography. Modern instruments provide an accurate representation of the anterior corneal surface and are used for corneal examination of normal and pathological shapes [1], contact lens fitting [2], or monitoring results of refractive surgery [3].

Classic videokeratometers often use single topography maps, obtained from the images of the Placido rings reflected from the cornea and the tear film. Although the instruments provide precise data [4], the cornea and the tear film change their properties with time [5-9]. Therefore, in some cases a sequence of images is needed to observe or measure variations of the corneal topography in time.

In the last few years, many authors have used dynamic videokeratometry to obtain better insight of the corneal surface and the tear film behaviour. Some of the classic static topographers can acquire at least one image per second which is stored in memory for later processing [10]. Although they provide useful information, measurement of the corneal and tear film dynamics requires much higher sampling frequency.

Commercially available systems provide reliable data; however many of these data are limited or controlled by proprietary software and researchers have not full control on all numerical processes.
In some applications [9-12], it could be interesting to access the raw data provided by the system or even implement new algorithms for data processing.

Sequences of the raw pictures of the Placido rings reflected from the cornea and the tear film can be used to observe fine dynamic variation of the corneal surface. These variations usually can not be observed using commercially available topographers since they give already processed data fitted to analytical surfaces, Some systems offer the possibility of saving pictures of the raw image [11], but control on the camera-exposition time and frame rate or potentiality replacing the $\mathrm{CCD}$ is limited or impossible in the vast majority of cases.

The Medmont E300 unit (Medmont Pty Ltd, Melbourne, Australia) can acquire images at a sampling frequency of $25 \mathrm{~Hz}$, allowing the analysis of the corneal dynamics. Recently, Iskander and Collins proposed a modification to a Medmont topographer providing a system capable of recording data at $50 \mathrm{~Hz}$. Higher sampling frequency allowed the study of the tear film stability and analysis of the dynamic response of the anterior corneal surface to the mechanical forces exerted by the eyelids during eye movements [11,12]. Although their innovative proposal allows accurate exploration of the corneal dynamics, no full access to the CCD parameters - gain, frame rate, resolution - is assured and the topography reconstruction is still done through the Medmont software.

*Corresponding author. Email: david.mas@ua.es

ISSN 0950-0340 print/ISSN 1362-3044 online

(C) 2009 Taylor \& Francis

DOI: $10.1080 / 09500340903050942$

http://www.informaworld.com 
It may be interesting for many researchers to apply their own elevation algorithms for measuring optical surfaces. Algorithms for reconstructing the corneal topography from the shape of reflected Placido ring images are available in the literature [13-15]. Nevertheless, these algorithms require precise knowledge of the geometry of the system projection head. Unfortunately, the design and exact geometry of such a projection head is also proprietary. Hence the user has almost no chance to test reconstructing algorithms.

The aim of this work is to demonstrate the transformation of a standard videokeratometer into a fully customizable device. A projection head from an existing device was used. All the imaging and electronic parts were dismounted and reassembled with our own video camera and computer. The system was calibrated with a set of steel calibration spheres. By using adequate image processing algorithms we are able to obtain the topography of the spheres and of the cornea. The camera can work as a static system, with one single capture, or in continuous video capture mode, which allows dynamic measurements of the corneal topography. The method of calibrating a videokeratometer using steel balls has been already implemented in [16], but the authors used a projection head with fewer Placido rings and less calibration balls which limited the obtained accuracy. Furthermore, the authors presented the methodology, but no quantitative results were given. To our knowledge, no improvements or further work on this method has been presented.

The method presented in this paper can be also used to extend the applications of commercial videokeratometers in the case when the access to recorded images is available. The Medmont E300 already provides enhanced and centred images of Placido reflections on the cornea that can be also used with some of the methods described below. All procedures and algorithms needed to construct a dynamic videokeratometric system by adapting the ring projection system are presented here. The obtained data proved to be consistent and comparable with results existing in the literature [17,18]. The construction of such a system provides accurate videokeratometric measurements and reduces limitation linked to commercially available systems.

\section{Experimental setup}

120 A custom-designed videokeratometer was constructed from a non-operative unit Humphrey Atlas 995 from Zeiss Meditec. The head consists of 22 rings with a peak in the near infrared. The camera and the computer were removed from the system and replaced by our own videocamera. In this case we have used a colour CMOS firewire camera Pixelink PL-A662 with $1 \mathrm{Mpx}$ of resolution. The camera has an infrared filter that has been removed to allow a better quality of the captured image. The naked CCD has been placed in the rear part of the head. The exact geometry of the head does not necessarily need to be known since we will recalibrate the whole system.

All parameters of the CCD (gain, shutter and saturation) were adjusted in order to obtain an optimum contrast between the background image of the eye and the bright rings. An artificial eye ball was used to adjust the position of the new CCD. Inside the topographer there is a single lens that has been displaced along the axis to allow correct focusing of the rings' image on the sensor plane. After fixing the camera to the head, the system was ready for calibration. This adjustment has to be done only occasionally because the parameters are maintained for a set of observers.

Seventeen steel spheres of known curvature radius with $\pm 10 \mu \mathrm{m}$ of accuracy were used to calibrate the system, following the procedure presented in [16]. The head of the topographer was locked in a central position and the spheres were aligned with the videotopographer on an optical bench. Milimetric axial positioning was necessary to assure optimal focus.

An image of each calibrating ball was processed according to the algorithms that are presented below. The position of each point of reflected rings was determined for all the balls. Since the curvature radius of the spheres is known, it is possible to calculate a set of calibration curves with the mean radial coordinate (in pixels) obtained for each point in one ring and its corresponding known curvature radius (in $\mathrm{mm}$ ). In Figure 1, the experimental curves and the linear fit adjustments that provide the final interpolation map

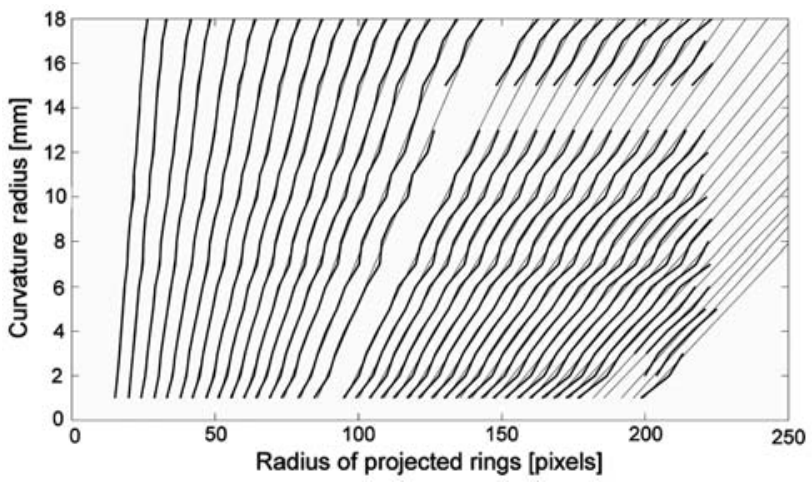

Figure 1. Interpolating curves representing correspondence between reflected rings' radii and curvature radii of the respective calibration spheres. Thick lines represent experimental data while thin lines represent the linear fits. 
are shown. Although positioning of the calibration spheres has been done carefully, there are some inaccuracies in the axial position of the system. The reason can be that even a small displacement of the system affects the image magnification. When it happens, all rings corresponding to a sphere of a given radius are incorrectly evaluated thus giving the wavy aspect to the figure. The effect is more noticeable for

170 the external rings where paraxial approximation is not accurate any more. Nevertheless, the linear fit compensates this effect and we can presume that the curvature radii are correctly evaluated.

The calculation of the curvature radius on a particular point of a curved surface (cornea) consists of two main steps. The first is a correct detection and classification of the ring, the second is an application of the interpolating curves [16].

\section{Image processing algorithms}

Focusing of the videokeratometer is carried out manually, so skilled operators are recommended for taking measurements. The video camera was controlled from an external computer. All frames were stored in the hard disk and afterwards processed with

\section{MATLAB software.}

Fine, fixation movements of the eye cause successive images of the Placido rings to be not centred at the same pixel. The first step in the registered image processing consists of adequate centring of the 190 topographies. Keratometers measure the topography of a surface with respect to a reference sphere so both the sphere and topographer have to be correctly aligned. Otherwise misalignments can produce aberrations, mainly prismatic effects and astigmatism that do

195 not come from the surface itself. Image alignment can compensate for such effects.

The image alignment is carried out as follows: a point inside the inner Placido disk of the first frame is manually selected. From this point, a vertical and a horizontal line are traced and intersection of both lines with the inner circle is calculated. The centre of the circle is determined by the intersection of the perpendicular bisector of these chords.

We would like to point out here that the topogra205 pher gives a 2D image of a three-dimensional structure and it is usually designed for working on spherical surfaces, so a centring algorithm may not be accurate for real eyes. Because the eye is not a sphere, the rings in the peripheral area may not be correctly centred.

210 Moreover, for large displacements of the eye the quality of the reflection is really poor and centring is not possible. In this case we are describing the decentration of the images which is really small (few pixels) and we are considering only the central ring, which is treated as a circle, so therefore the approximation is correct. Only in the case of large astigmatisms the central ring may look like an ellipse and other geometrical approaches should be considered.

Once the centre of the first frame has been determined, all remaining frames will be aligned with this one by using cross-correlation between each image and the first frame. The correlation is done in a central area of $100 \times 100$ pixels, which include the first four rings of the topography and the reflection from the fixation point. During the measurement the eye pupil is always large enough, so the analyzed rings are always located on the dark background. Centred images are then cropped to the region of interest. In Figure 2(a) the appearance of the preprocessed image of Placido rings' reflection after the centring procedure is shown. It is worth noting here that the centring procedure also provides data for the transversal movements of the corneal apex, which can be useful in some applications of eye movement examinations.

Curvature analysis is easier to do in polar coordinates since one can take advantages from the symmetry of the rings (see Figure 2(b)). Cartesian to polar coordinates requires an accurate calculation of the centre of the image. If this centre is not well chosen, the rings will appear as wavy lines. Conversely, if the frame is correctly realigned, the central rings will appear as straight lines, and thus, image and numerical analysis is easier.

Detection of the Placido rings consists of detecting the border points of all transitions between dark and bright areas. In [19] the authors compare different procedures for extracting these borders. In our case a Canny filter was used. The Canny edge detector [20] uses a filter based on the first derivative of a Gaussian function. The method can be summarized in two steps consisting of noise cleaning by Gaussian blur and then calculating the gradient of the filtered image. The borders of the image are identified as the peaks of the absolute value of the gradient map. The method is fast and efficient and its computational implementation can be found in different mathematical packages.

In Figure 3, the result of the Canny filter applied on the image in Figure $2(b)$ is presented. Notice that the majority of the borders are detected, but there appears to be some noise and false vertical borders, due to the presence of eyelashes and shadows. Most of this noise can be filtered by applying horizontal filters like median filters or morphological erosions [21]. In $[9,11]$ the authors use a similar technique to study the quality of the tear film. They use images similar to that in Figure 3 and analyze the structure of the lines to explore the tear film surface quality. 

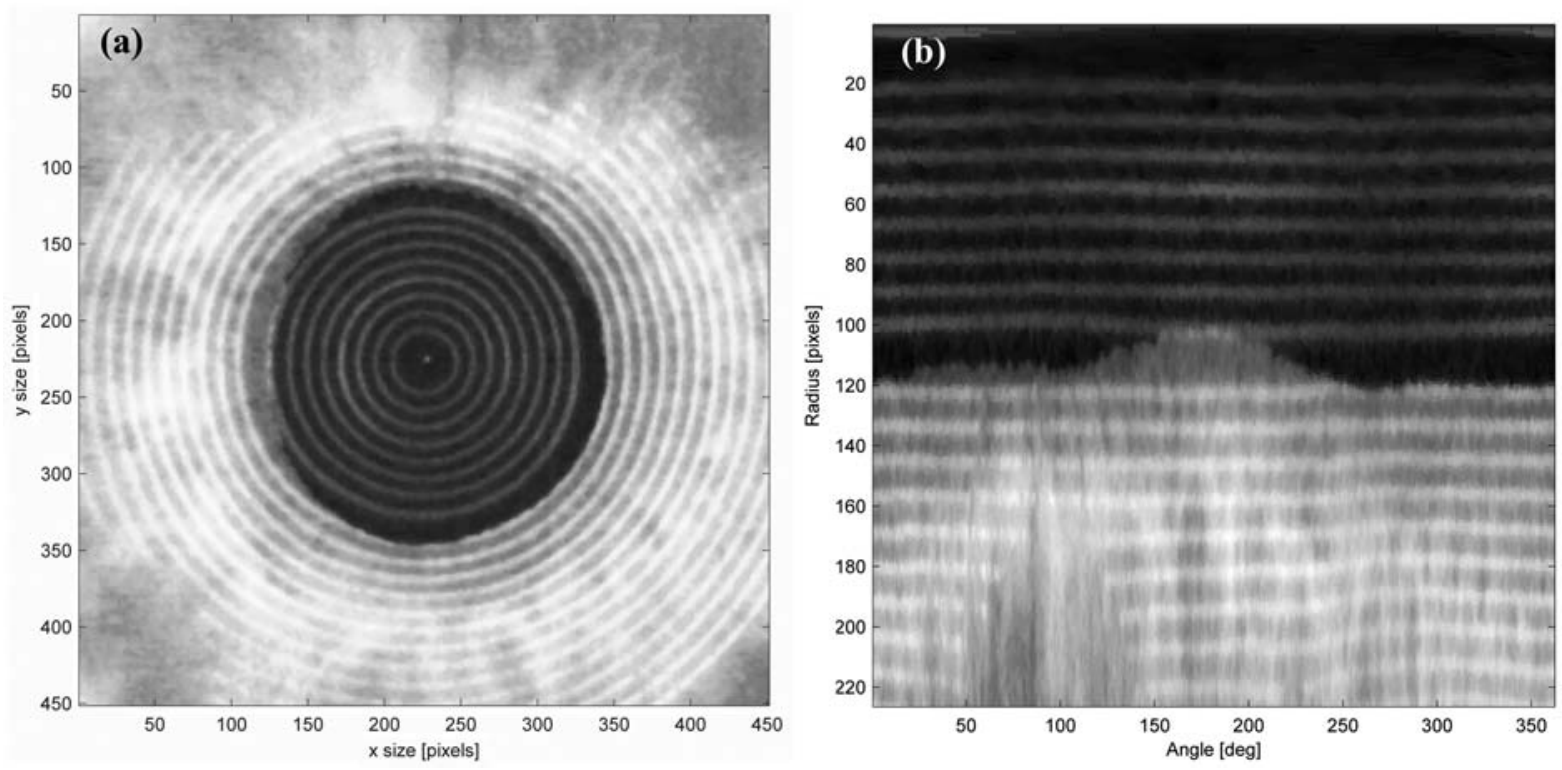

Figure 2. (a) Picture of the Placido rings reflected from the cornea after cropping and centring the region of interest. (b) Image of Placido rings in polar representation. Vertical and horizontal axis represent radial and angular coordinates.

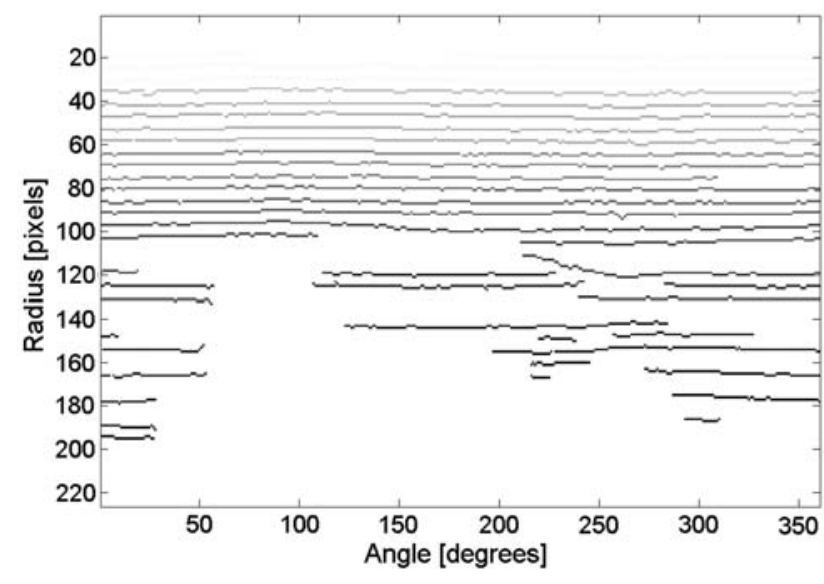

Figure 3. Result of a Canny filter applied on Figure 2(b) (negative image). Notice the presence of diagonal and vertical borders due to the pupil and eyelashes. Different gray levels correspond to different labelled rings.

Each detected ring is classified and labelled with a cardinal number. Correct labelling is the critical step in all the procedure due to the fact that the selection of the correct interpolation curve in Figure 1 depends on the labelling. The label is first assigned at the first column of Figure $3\left(0^{\circ}\right)$ and then the line path continuity is followed through the image. Gaps in the ring interrupt the labelling process, so the procedure is repeated starting from different columns.

An important source of error comes from misclassification of the rings due to two reasons. On the one hand, vertical connection between rings means that two successive rings are classified with the same label.
On the other hand, the absence of a ring means that two non-consecutive rings are classified with consecutive labels. Both errors can be avoided by considering the radius of the ring. If the same label appears for two different distances, the ring is not taken into account for the calculation. In Figure 3 the image of the rings after labelling is presented.

After classification of the rings, a curvature radius has to be assigned to each labelled point. The procedure was first applied to the steel balls where the curvature is known, and the interpolation curves in Figure 1 were obtained. Afterwards, for the cornea, the procedure is repeated. Each labelled ring corresponds to one interpolation curve and the radius of curvature of each point in the ring is obtained. From the curvature data, one can easily obtain the topography of the surface that can be smoothed by fitting Zernike polynomials.

\section{Calibration results}

Prior to making any ocular measurement the consistency of the method was checked. With the calibrated device the curvature radius of each sphere mounted on the optical bench was measured again. In Figure 4 the results of such measurements are presented and we can see the good reproducibility of the algorithm $\left(r^{2}>0.99\right)$.

The tolerance to the axial displacement was also checked. Axial displacement of the surface produces a different lateral magnification of the optical system so the curvature radius is not evaluated correctly. 


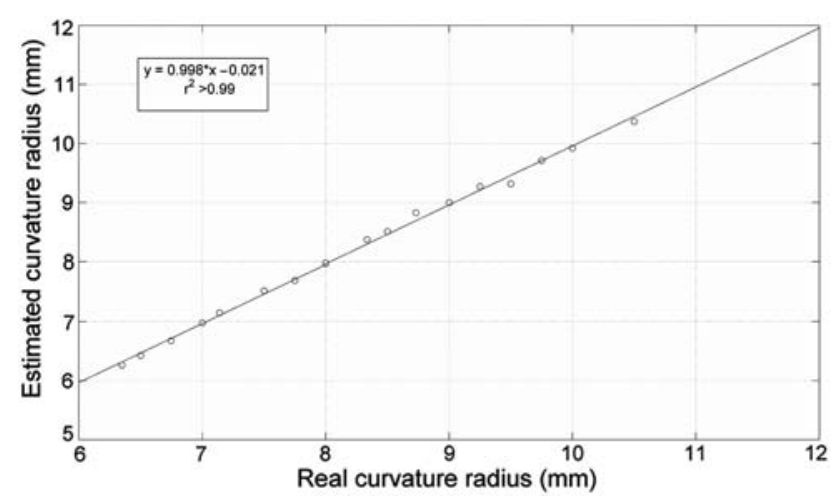

Figure 4. Proof of consistence of the method. Correspondence between real curvature radius and measured curvature radius for the calibrating spheres for a pupil radius of $4.03 \mathrm{~mm}$.

We placed on the optical bench a steel sphere of $8 \mathrm{~mm}$ of curvature radius and displaced it back and forth in steps of $0.5 \mathrm{~mm}( \pm 0.1 \mathrm{~mm})$ in the range of $\pm 1.5 \mathrm{~mm}$

315 around the optimal position. Images of the sphere were captured and processed. In Figure 5 we present the calculated radius of curvature as a function of the defocused distance calculated, for the rings within an area of $8 \mathrm{~mm}$ of diameter.

From Figure 5 one can see, that $1 \mathrm{~mm}$ of axial displacement of the surface produces an error in the estimation of the curvature of $0.3 \mathrm{~mm}$. Let us consider now an ideal cornea as a spherical surface with $8 \mathrm{~mm}$ of curvature radius that separates air of a medium of index $n=1.3375$. Such a surface has an optical power of 42.2D. Standard corneal topographers have a tolerance of $\pm 0.2 \mathrm{D}$, or equivalently around $0.04 \mathrm{~mm}$ in the curvature radius. Therefore, these systems can tolerate an error in the position of the eye of $0.13 \mathrm{~mm}$.

\section{Dynamic corneal measurements}

Once the system was calibrated, we proceeded to measure the corneal topography. Since the system allows continuous recording at a video rate, we performed corneal measurements for three subjects 335 during $10 \mathrm{~s}$ (HK, man aged 60, MK, woman aged 26 and MD aged 24), at a frame rate of $28 \mathrm{~Hz}$. The subjects were placed in a head rest with a bite bar and were asked to suppress blinking during the measurement. We measured both eyes for subjects HK and MD and only the left eye for the subject MK.

Notice that almost all chinrests attached to topographic devices are not rigid enough and allow displacements of the head, thus inducing some errors in the estimation of the curvature radius. This error can increase significantly if the patient does not vave

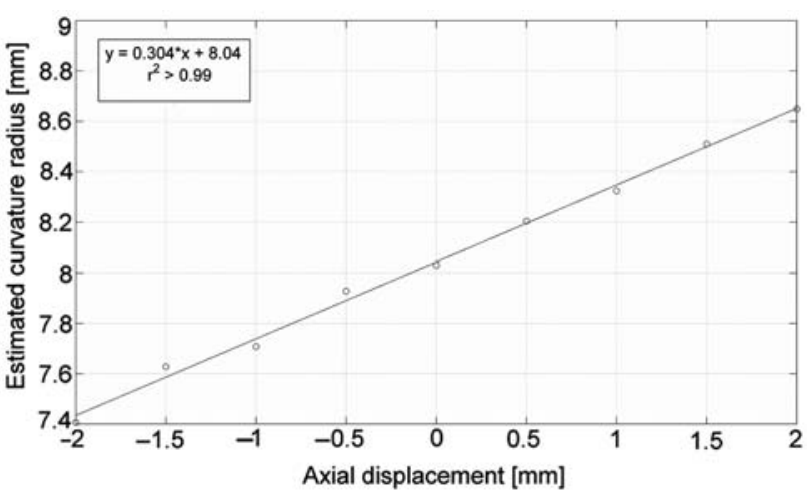

Figure 5. Tolerance to axial displacement. Axial displacement provokes diffuse ring borders, changing magnification and errors in the estimation of the curvature.

source of errors in the topographers, but it is clear that axial position is a factor that can limit the accuracy of the device.

Here, head movements were recorded with a custom-designed air-coupled ultrasound transducer working at $0.8 \mathrm{~Hz}$ (Medicom S.C., Wroclaw, Poland.), which allowed one to measure the normal displacements with an accuracy of $2 \mu \mathrm{m}$. The subjects were asked to breath with a metronome, so respiratory rhythm was set to $0.5 \mathrm{~Hz}$. From the five series processed, we will only present here data from the left eye of the observer HK (HK-OS). For the other observers results are comparable so they will not be depicted.

From obtained height data, we have reconstructed the surface and the main optical descriptors: i.e. spherical power $(M)$ and regular and oblique astigmatism, through the vectors $\mathrm{J} 0$ and $\mathrm{J} 45$ [22]. Each subject that participated in this experiment was also measured with a Pentacam device, so a reference corneal topography was obtained.

We would like to emphasize that correct centering and focusing of the image is done at the first frame but after that the system records images automatically. During the $10 \mathrm{~s}$ sequence the head and the eye are moving. Along the process, there is no control about changing magnification or image blurring. All the frames are processed by the computer and only at the end of the procedure one can check the result. All points in the final processed matrices that are correctly detected are assigned to their height values, while those points where ring detection failed have been labelled with negative height value. We also labelled all those points whose obtained height is outside some local defined bounds. With the valid points, the curvature is reconstructed and interpolated for all of the surfaces points. Results obtained (Zernike coefficients, curvature values, astigmatism,...) are his head well positioned. Little attention is paid to this 
written in a vector form and treated as a time series. Noise and outliers were filtered with a median filter of order 3.

In Figure 6, we present the point-to-point median difference between the reference topography recorded by Pentacam and one set of topographies measured for the left eye of observer HK with our dynamic topographer. The shown area corresponds to a pupil diameter of $4 \mathrm{~mm}$. The mean value of the absolute difference is $1.0 \pm 0.8 \mu \mathrm{m}$ (mean $\pm \mathrm{SD})$. Notice that the main errors come from the lower part. For another data series registered on the same eye or on eyes of other subjects, we have observed that larger errors usually appear in the lower or upper part of the map This can be an effect of the eyelids presence or irregularities in the tear film. Regarding the central part of the surface, for all subjects we have obtained the same error distribution, i.e. clear difference between the upper and the lower part of the map. This suggests that the main source of error comes from optical misalignment between the eye and the camera or differences between head positions on both devices, which may induce some tilt in the measured surface. Although tilt is not taken into account in the aberration analysis, misalignment between the eye and the topographer optical axis may induce the presence of small coma or astigmatism by oblique incidence.

From elevation data, we have obtained the values of spherical and astigmatic components. In Figure 7, we show the values obtained for the $M$ component together with the reference value. It can be seen that reproducibility of our device is relatively good since the reference value for this eye is $44.4 \mathrm{D}$ while we have

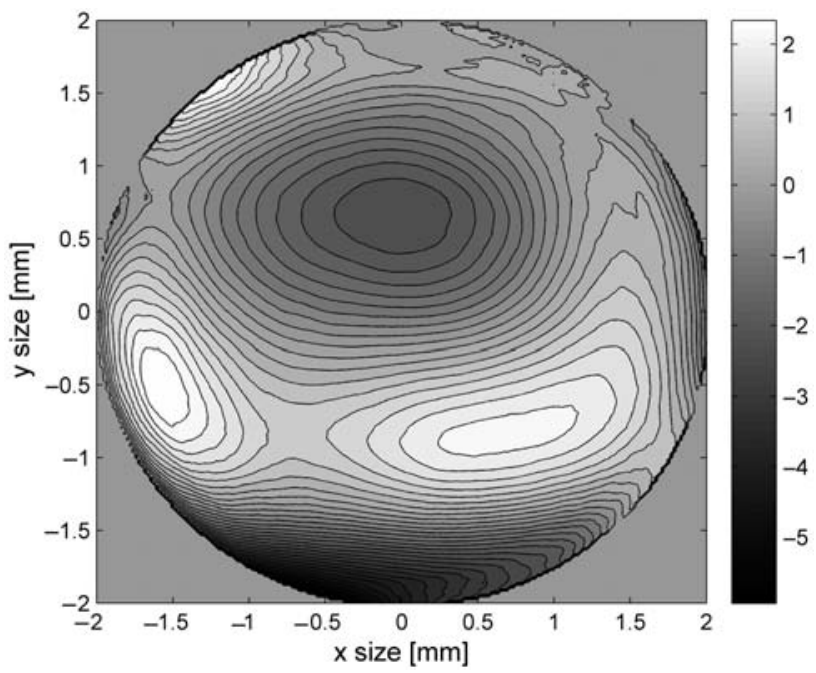

Figure 6. Mean difference between all topographic maps obtained for the left eye of subject HK (280 frames) and a reference topographic map taken with Pentacam. Difference values in the colour bar are given in $\mu \mathrm{m}$. obtain a mean value of $43.3 \pm 0.6 \mathrm{D}$. Very fast variations of the amplitude around $1 \mathrm{D}$ can be noticed. As explained above lateral displacement has been removed, but not the axial displacement. According to the figure, head movement in this direction oscillates around $20 \mu \mathrm{m}$, so this will give a variation of around $0.15 \mathrm{D}$.

In [23] the authors report a change also in the best fit sphere that can reach even $120 \mu \mathrm{m}$, depending on the subject so giving oscillation of about 1D. As can be seen in Figure 8, most repeated oscillations are of about $0.5 \mathrm{D}$ and may probably come from head movements. Larger variations in such small time are probably due to noise and should be filtered out. These peaks can be seen in results obtained for all subjects. The corresponding Placido images were sometimes blurred or displaced, probably due to the fast saccades and thus our software cannot process them correctly. We would like to emphasize that an additional advantage of our proposal is that the blur can be controlled by the shuttering time of the CCD provided that a fast enough device is available. Moreover, commercial topographers do not analyze the reflected image if a significant area of the Placido rings while in our proposal this limitation can be avoided.

We have found that the power of the spherical component measured with Pentacam is, on average, $0.5 \mathrm{D}$ higher than that obtained with our dynamic topographer. For one of the subjects participating in the experiment (MD), the difference of the mean spherical power was evenly equal around 1.7D. If we suppose that the estimation error of the Pentacam and topography devices is of about $0.2 \mathrm{D}$, the difference would be up to $0.4 \mathrm{D}$, so the deviation of our results can be considered moderate. In any case, the fact that all except one of our results are systematically below those

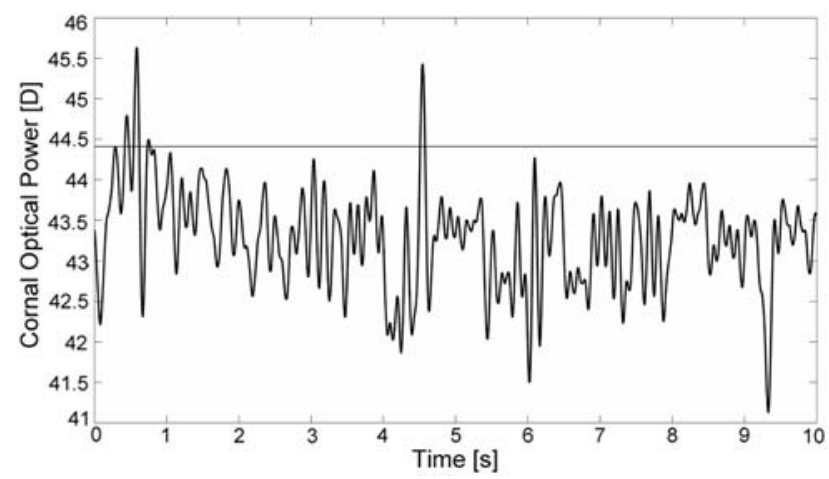

Figure 7. Measured values of spherical component of the cornea for the left eye of subject HK. Straight line represent mean spherical component measured with Pentacam (44.4D) while rough line represents the temporal sequence (mean value $43.3 \pm 0.6 \mathrm{D})$ 
of the Pentacam, means that a systematic error is 455 present. Such error may be due to an axial misplacement of the head or the effect of the possible systematic error of the Pentacam. Further analysis with a larger number of subjects is required before stating a clear conclusion.

To show the influence of the head movement on the measured optical power, we presented in Figure 8 the optical power $M$ and synchronously registered head movement for subject HK-OS. To facilitate comparison, both data have been linearly detrended.

465 Mean amplitude of the head oscillation is around $20 \mu \mathrm{m}$. According to our calculation in the previous section this movement induces an error of about 0.06 $\mathrm{mm}$ in the curvature radius, or equivalently $0.15 \mathrm{D}$, which is around the nominal power resolution of a standard videokeratometer. Therefore the peaks in the head movements are not automatically translated into peaks in the spherical power although the influence is clear. The slow trend observed in the corneal power variations is clearly the effect of the head movement.

Mean oscillations of the optical power observed for all subjects were much higher than those that were coming from the head movement. From Figure 1, one can deduce that small variations of the curvature radius induce important changes in the ring position. It has been reported in the bibliography that the cornea pulsates with cardiopulmonary rhythms [23]. Fourier analysis has been performed on the temporal series of $M$, but in all cases the frequency spectrum was too noisy to find any clear component except respiration. presence of the heart pulse. We expect that by using a camera of higher temporal and spatial resolution we could reveal these expected relationships.

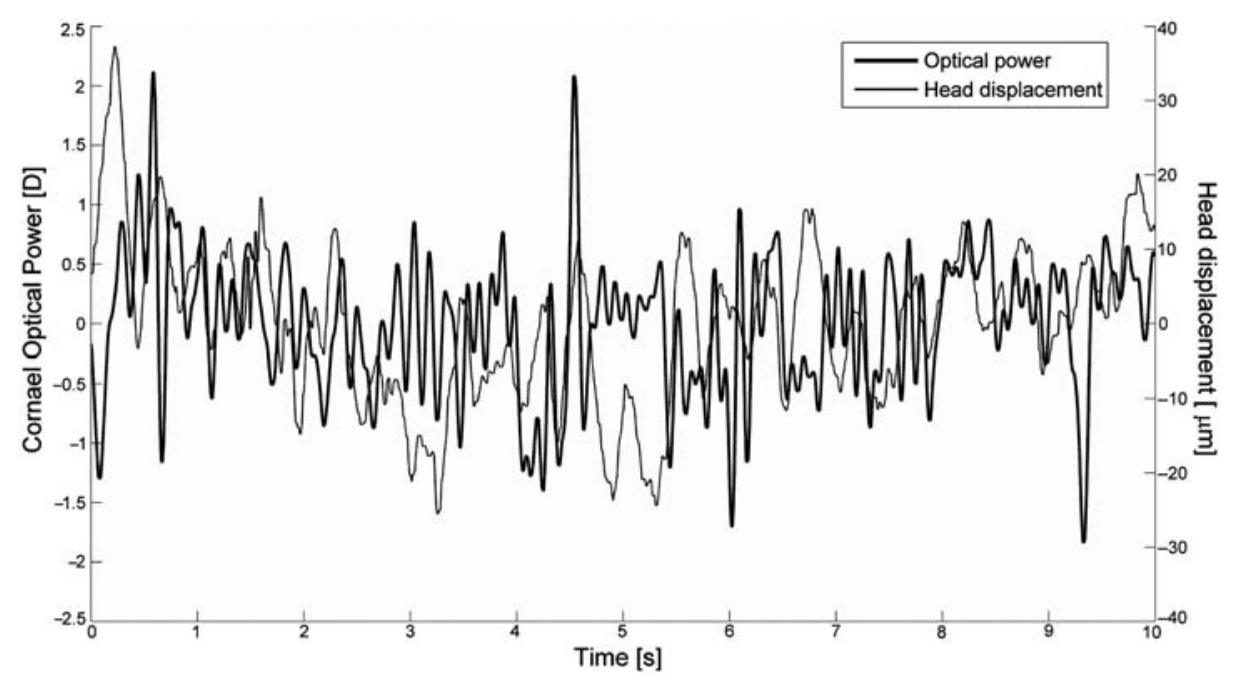

Figure 8. Measured values of spherical component of the cornea for the left eye of subject HK and head movements for the same subject. Both graphs are linear detrended.

In Figure 9 variations of the $\mathrm{J} 0$ and $\mathrm{J} 45$ astigmatic components, registered for the subject HK-OS are shown. The oscillation of these components is very high. For $\mathrm{J} 0$ the reference obtained form Pentacam value is of $-0.4 \mathrm{D}$ while we obtain mean value of $-1.0 \pm 0.8 \mathrm{D}$. In the case of oblique astigmatism, reference value is $-0.0 \mathrm{D}$ in comparison to $0.9 \pm$ $0.8 \mathrm{D}$. In general, measurement of astigmatism by our system is less accurate than those of spherical components. There is an additional source of error since, as it was pointed before in case of Figure 6, the estimation of the surface can be affected by some tilt and this fact may affect the polynomial fit.

From the values of $\mathrm{J} 0$ and $\mathrm{J} 45$ it is possible to obtain the orientation of the axis of astigmatism [24]. Unfortunately, the subject $\mathrm{HK}$ and $\mathrm{MD}$ have a subjective astigmatism of less than $0.5 \mathrm{D}$, which is on the limit of the resolution of measuring devices. Only the left eye of the subject MK had an astigmatic cornea. The data obtained in this case was of $19^{\circ} \pm-8^{\circ}$ in comparison to a reference of $31^{\circ}$. This oscillation is slightly higher than that presented in the literature. Ferman et al. [17] mentioned oscillations from $1.5^{\circ}$ to $5^{\circ}$ while a subject was looking into a central target, Buheren et al. reported a mean oscillation of $2.4^{\circ} \pm 1.1^{\circ}$ [18]. In any case it is difficult to obtain any significant conclusion with results obtained only for one subject. In any case, axis of astigmatism is directly obtained from $\mathrm{J} 0$ and $\mathrm{J} 45$ and these data have been already discussed.

The obtained results can be used for eye dynamics analysis, but also for static measurements. Common protocols consist of taking three or more images and average results. With the presented system one can capture a video sequence of a few seconds and 


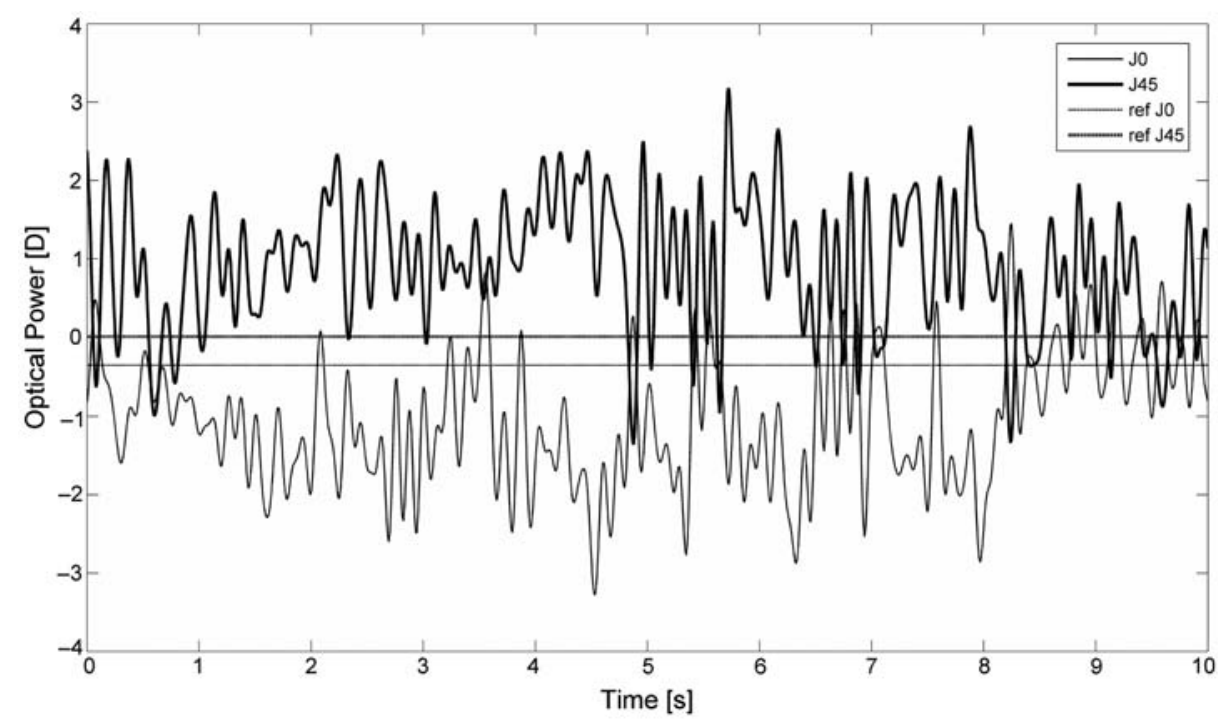

Figure 9. Measured values of J0 and J45 astigmatic components for the left eye of subject HK. Reference value for J0 is $-0.4 \mathrm{D}$ while mean value obtained with the dynamic topographer is $-1.0 \pm 0.8 \mathrm{D}$. Reference value for J45 is $0.0 \mathrm{D}$ while mean value obtained with the dynamic topographer is $0.9 \pm 0.8 \mathrm{D}$.

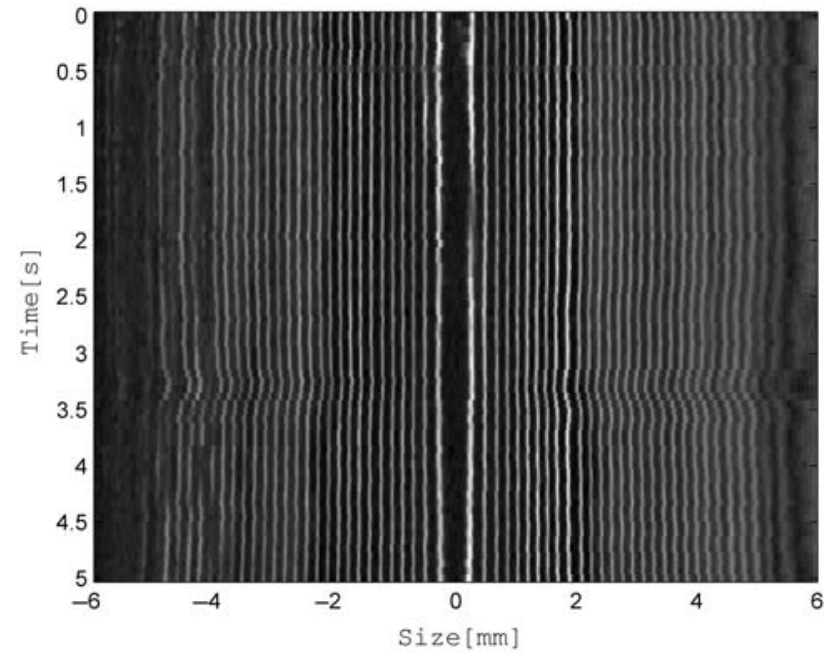

Figure 10. Sequence of the central meridian of a corneal topography. Notice the contraction at second 3.5 due to a suppressed blink.

average data. Depending on sampling frequency of the camera, it can improve the accuracy of the measurement with respect to the static measurement.

The analysis of the raw images of the reflected Placido rings can provide useful information about cornea dynamics. In Figure 10 we present a profile view of the temporal variation of the horizontal meridian of the image. At $3.5 \mathrm{~s}$, a corneal contraction due to a suppressed blink can be observed. This contraction can be hardly seen in a surface analysis, but from this picture it is very clear.

\section{Conclusion}

Commercial videokeratometers provide useful information about corneal topography and the tear film quality. Some of them are capable of acquiring dynamic sequences of the eye, but none allow full control of the camera and processing algorithms.

We have presented methods and procedures to build a dynamic topographer from an exiting videokeratometer projection head and a video camera. Algorithms for processing the images have also been detailed. We have presented the calibration process and the procedures to reconstruct the curvature data. Some results and applications also have been outlined.

The obtained results are limited by the calibration procedure and applied algorithms. The numerical processing of the images is complex and many small variations are averaged and lost in the process. Nevertheless, if one is interested in relative variations, our results show that there is much information hidden in the process of obtaining a corneal topography, and many of these data can be of great interest for researchers. From the algorithms here presented it is possible to introduce convenient modifications in the calibrating process, temporal and spatial resolution of the camera and the numerical algorithms to obtain new information about the dynamic processes of the cornea and the tear film.

Open access to the software and to the parameters of the videocamera permits more complex experiments that those that can be done with a commercial device. With an adequate camera, one can change the capturing speed, integration time, resolution of the 
CCD and many other parameters that may enhance the acquired images. Additionally one can design its own software to select the number of rings to analyze, focus the analysis to a particular region of interest and apply different image processing algorithms to obtain data from the structure reflected on the cornea. Recently, Alonso-Caneiro et al. [25] presented an algorithm for estimating corneal surface topography in the presence of strong noise. This procedure or any other can be added to the algorithms presented in this work in order to produce more accurate results and further exploration of the corneal dynamics.

\section{Acknowledgements}

580 This work has been partially supported by the Spanish Ministry of Education and Science through the project no. FIS2005-05053. H. Kasprzak acknowledges the University of Alicante its economical support through the project Senior 08/07. D. Mas acknowledges the support of the Generalitat Valenciana through the project BEST-2008-148.

\section{References}

[1] Schwiegerling, J.; Greivencamp, J.E. Optom. Vis. Sci. 1996, 73, 721-728.

[2] Szczotka, L. Optm. Vis. Sci. 1997, 74, 14-19.

[3] Holladay, J.T.; Dudeja, D.R.; Chang, J. J. Cat. Ref. Surg. 1999, 25, 663-669.

[4] Jeandervin, M.; Barr, J. Optom. Vis. Sci. 1998, 75, 663-669.

[5] Buheren, T.; Collins, M.J.; Iskander, D.R.; Davis, B.; Lingelbach, B. Cornea 2001, 20, 826-833.

[6] Erdelyi, B.; Csakany, B.; Rodonyi, G.; Soumelidis, A.; Lang, Z.; Nemeth, J. Ophthal. Physiol. Opt. 2006, 26, 419-425.

[7] Zhu, M.; Collins, M.J.; Iskander, D.R Eye 2007, 21, 624-632.
[8] Nemeth, J.; Erdelyi, B.; Csakany, B.; Gaspar, P.; Soumelidis, A.; Kahlesz, F.; Lang, Z. Inv. Opth. Vis. Sci. 2002, 43, 1783-1790.

[9] Kopf, M.; Yi, F.; Iskander, D.R.; Collins, M.J.; Shaw, A.J.; Straker, B. J. Optom. 2008, 1, 14-21.

[10] Goto, T.; Zheng, X.; Klyce, S.D.; Kataoka, H.; Uno, T.; Karon, M.; Tatematsu, Y.; Bessyo, T.; Tsubota, K.; Ohashi, Y. Am. J. Ophthal. 2003, 135, 607-612.

[11] Iskander, D.R.; Collins, M.J.; Davis, B. IEEE Trans. Biomed. Eng. 2005, 52, 1939-1949.

[12] Iskander, D.R.; Collins, M.J. Clin. Exp. Optom. 2005, I, 223-231.

[13] Klein, S.A. Optm. Vis. Sci. 1992, 69, 829-834.

[14] Klein, S.A.; Mandell, R.B. Inv. Opth. Vis. Sci. 1995, 36, 2155-2159.

[15] Mattioli, R.; Tripoli, N.K. Optom. Vis. Sci. 1997, 11, 881-894.

[16] De Carvalho, L.A.V.; Romao, A.C.; Tonissi, S.; Yasuoka, F.; Castro, J.C.; Schor, P.; Chamon, W. Arq. Bras. Oftalmol. 2002, 65, 37-41.

[17] Ferman, L.; Collewijn, H.; Jansen, T.C.; Van den Berg, A.V. Vision Res. 1987, 27, 811-828.

[18] Buehren, T.; Lee, B.J.; Collins, M.J.; Iskander, D.R. Cornea 2002, 21, 346-351.

[19] De Carvalho, L.A.V.; Bruno, O.M. Comput. Meth. Programs Biomed. 2005, 79, 111-119.

[20] Canny, J.F. IEEE Trans. Pattern Anal. Machine Intellig. 1986, 8, 679-698.

[21] Serra, J. Image Analysis and Mathematical Morphology; Academic Press: Orlando, FL, 1983.

[22] Iskander, R.; Davis, R.A.; Collins, M.J.; Franklin, R. Ophthal. Physiol. Opt. 2007, 27, 245-255.

[23] Iskander, D.R.; Kasprzak, H.T. Ophthal. Physiol. Opt. 2006, 26, 572-579.

[24] Salmon, O.T.; West, R.W.; Gasser, W.; Kenmore, T. Optom. Vis. Sci. 2003, 80, 6-14.

[25] Alonso-Caneiro, D.; Iskander, D.R.; Collins, M.J. IEEE Trans. Biom. Eng. 2008, 55, 2381-2387.

[26] Kasprzak, H.T.; Iskander, D.R. IEEE Trans. Instrum. Meas. (accepted). 